\title{
Perceptual anticipation in handwriting: The role of implicit motor competence
}

\author{
SONIA KANDEL \\ University of Geneva, Geneva, Switzerland \\ and Pierre Mendès-France University, Grenoble, France \\ JEAN-PIERRE ORLIAGUET \\ Pierre Mendès-France University, Grenoble, France \\ and \\ PAOLO VIVIANI \\ University of Geneva, Geneva, Switzerland \\ and UHSR University, Milan, Italy
}

\begin{abstract}
In two experiments, perceptual anticipation-that is, the observer's ability to predict the course of dynamic visual events-in the case of handwriting traces was investigated. Observers were shown the dynamic display of the middle letter $l$ excerpted from two cursive trigrams ( $l l l$ or $l l n$ ) handwritten by one individual. The experimental factor was the distribution of the velocity along the trace, which was controlled by a single parameter, $\beta$. Only for one value of this parameter $(\beta=2 / 3)$ did the display comply with the two-thirds power law, which describes how tangential velocity depends on curvature in writing movements. The task was to indicate the trigram from which the trace was excerpted-that is, to guess the letter that followed the specific instance of the $l$ that had been displayed. In Experiment 1 , the no answer option was available. Experiment 2 adopted a forced-choice response rule. Responses were never reinforced. When $\beta=2 / 3$, the rate of correct guesses was high (Experiment $1, P$ [correct $\}=$ .69 ; Experiment $2, P[$ correct $]=.78$ ). The probability of a correct answer decreased significantly for both smaller and larger values of $\beta$, with wrong answers becoming predominant at the extremes of the range of variation of this parameter. The results are consistent with the hypothesis that perceptual anticipation of human movements involves comparing the perceptual stimulus with an internal dynamic representation of the ongoing event.
\end{abstract}

Biological motion - that is, voluntary movements of the body-has peculiar perceptual qualities acknowledged and investigated since the beginning of the century (Kenkel, 1913; Korte, 1915; Wertheimer, 1912). Salience is one striking feature of biological motion: Even very sketchy descriptions of whole-body movements can be detected (Johansson, 1950) and discriminated (Beardsworth \& Buckner, 1981) accurately, within a few hundred milliseconds (Johansson, 1973; Johansson, Von Hofsten, \& Jansson, 1980). Such a high level of perceptual tuning is already present in 5-month-old babies (Bertenthal, Proffitt, \& Cutting, 1984; Bertenthal, Proffitt, \& Kramer, 1987) and actually is likely to be an innate endowment. Indeed, the ability to single out biological motion from other types of physical motion may have adaptive value, inasmuch as it provides the basis for

This work was partly supported by a research grant to P.V. from the Pôle Rhône-Alpes des Sciences Cognitives. We are grateful to Ruud Meulenbroek and to two anonymous reviewers for their comments and criticisms. Correspondence concerning this article should be addressed to P. Viviani, Department of Psychobiology, Faculty of Psychology and Educational Sciences, University of Geneva, 9, Route de Drize, 1227 Carouge, Switzerland (e-mail: viviani@uni2a.unige.ch). anticipating future behavior (Cooper, 1992; Freyd, 1993; Prinz, 1992).

Arguably, the perceptual salience of biological motion is rooted in the implicit knowledge (Palmer, 1978) that humans seem to have about their own body and movements. Evidence for this claim is provided by a number of interaction effects. For instance, still pictures of the human body displayed sequentially, with appropriate spatiotemporal parameters, give rise to the phenomenon of apparent motion (Korte, 1915). However, perceptual solutions that would normally be privileged (viz., the shortest-path movement that, according to Korte's law, is perceived whenever several paths are possible) are not selected if they are in conflict with biomechanical constraints (Shiffrar \& Freyd, 1990, 1993). Similar interactions are also present in the cases of movements about inanimate objects (Heptulla Chatterjee, Freyd, \& Shiffrar, 1996) and walking movements (Thornton, Pinto, \& Shiffrar, 1998), suggesting that the selection of one perceptual solution among several alternatives is biased by knowledge of human motor limitations.

Another class of motor-perceptual interactions arises in conjunction with an empirical motoric rule known as the two-thirds power law (Lacquaniti, Terzuolo, \& Viviani, 
1983; Viviani \& Schneider, 1991; Viviani \& Terzuolo, 1982), which applies to most end-point voluntary movements (e.g., drawing and writing). In general, the tangential velocity of a smooth point movement can be specified independently of the shape of its trajectory, the only constraint being that velocity must go to zero at cusps. Endpoint voluntary movements, however, are special in that, at any regular point of the trajectory, the tangential velocity and the curvature of the trajectory are inversely related in a precise manner specified by the two-thirds power law (see the Method section). This covariation is one of the qualitative attributes that set biological motions apart from most artificially generated motions. The visual system deals more effectively with stimuli that have this particular attribute than with stimuli that do not. The geometric (Viviani \& Stucchi, 1989) and kinematic (Viviani \& Stucchi, 1992) properties of dynamic two-dimensional (2-D) point-displays are misjudged when the curvaturevelocity relationship does not comply with the power law, as it would if the display depicted a real voluntary movement. Moreover, the accuracy of visuomanual (Viviani, Campadelli, \& Mounoud, 1987; Viviani \& Mounoud, 1990) and oculomotor (de'Sperati \& Viviani, 1997) 2-D tracking depends on the extent to which the target's movement complies with the power law. This peculiar sensitivity is not limited to vision. A recent study (Viviani, Baud-Bovy, \& Redolfi, 1997) showed that passive arm movements that violate the power law cannot be either perceived kinesthetically or reproduced accurately.

The findings summarized above suggest that the intuition underlying Liberman's motor theory of speech perception (Liberman, Cooper, Shankweiler, \& StuddertKennedy, 1967; Liberman \& Mattingly, 1985) may be generalized to nonlinguistic stimuli by postulating that perceptual representations of biological motion are always identified and analyzed with the contribution of functional modules embodying our motor competence. Assuming the validity of this generalization, we address here the further question of whether motor competence is also involved in perceptual anticipation-that is, in the ability to predict the future course of dynamic perceptual events.

Planning and execution of complex sequences of movements involve a significant amount of look-ahead. In fact, units of motor action being executed often carry the imprint of yet-to-be-executed units. These anticipatory adjustments, which are due to coarticulation, are well documented in movements such as speech (Benguérel \& Cowan, 1974), typing (Viviani \& Laissard, 1996), and handwriting (Thomassen \& Schomaker, 1986; Van Galen, Meulenbroek, \& Hylkema, 1986). In particular, the kinematics of a letter in cursive handwriting varies according to the size, shape, and direction of rotation of the following letter (Van Galen et al., 1986). Clearly, some of the contextual variations affecting the ongoing action provide clues on what the forthcoming action will be. It has been shown (Kandel, Orliaguet, \& Boë, 1994; Orliaguet,
Kandel, \& Boë, 1997) that, by viewing only a dynamic display of the letter $l$ while it is being written, observers can reliably predict the identity of the following letter well before transition cues become available. This specific form of perceptual anticipation was demonstrated both with isolated digrams and with digrams embedded within words, irrespective of writing size or mean velocity (Kandel, Boë, \& Orliaguet, 1993; Kandel, Orliaguet, \& Boë, 1995). Total duration does not seem to provide a discriminating cue. In digrams such as $l l, l e$, and $l n$, whatever the total movement time, the second letter can be predicted reliably by viewing only a constant fraction (the final $60 \%$ ) of the downstroke of the $l$ (Kandel, 1995).

Here, we report a further study of perceptual anticipation in handwriting that extends the aforementioned research along two lines. Our first aim was to show that the relevant cues in this case are kinematic. Although coarticulation may manifest itself in subtle context-dependent variations of the shape of the movement (Orliaguet et al., 1997), we wanted to demonstrate that anticipation is not trivially based on the ability to discriminate these geometrical factors. Thus, the geometrical features of the dynamic stimuli were kept invariant across conditions. Our second and more important aim was to show that the perceptual system can get full access to the discriminating cues only when the dynamic trace complies with the curvature-velocity relationship that makes it a plausible representation of a biological motion. In turn, this would strongly suggest that, indeed, perceptual anticipation draws on implicit motor competence. The study combines the task designed by Kandel et al. (1994) with the experimental strategy adopted in the study by Viviani and Stucchi (1992). As in the former study, the participants had to guess the third letter of a trigram after seeing a dynamic display of just the second one, which was always the same. Moreover, as in the study by Viviani and Stucchi (1992), the stimuli differed only in the way tangential velocity varied along their trajectory, which was selected within a oneparameter family of velocity distributions. Only one of these distributions satisfied the two-thirds power law, making the display a plausible instance of biological motion. All other distributions represented graded departures from this natural motoric rule. The two aims of the study would be fulfilled by demonstrating that the ability to take advantage of coarticulatory cues for predicting the third letter decreases pari passu with the extent to which the tangential velocity distribution differs from the natural one.

\section{METHOD}

\section{Participants}

Forty-four right-handed students at Grenoble University participated in the experiment on a voluntary basis. The participants were between 23 and 28 years of age and had normal or corrected-tonormal vision. They were naive as to the purpose of the experiment. Twenty-two participants were tested with a three-choice response rule. The remaining 22 were tested with a forced-choice response rule. 


\section{Apparatus}

The experiment was run in a soundproof room kept in dim light. The participants were seated at a comfortable viewing distance from a 14-in. computer screen (Vx Macintosh). The display was controlled by a HyperCard 2.2 environment with a Pascal extension (XFCN). Responses were entered through the computer mouse.

\section{Stimuli and Task}

The stimuli were dynamic point-displays of cursive letters derived from samples of handwriting generated by one right-handed individual who did not participate in the experiment. The point left a trace on the screen that remained visible until the end of the movement. The first step in the generation of the stimuli was the recording and selection of a set of instances of the trigrams $l l l$ and $l n$. Handwriting traces were recorded with a digitizing tablet (Numonics Corporation, Montgomeryville, PA, Model 2002; sampling frequency, $200 \mathrm{~Hz}$; nominal resolution, $0.02 \mathrm{~mm}$ ). Two horizontal lines $6 \mathrm{~cm}$ apart delimited the vertical extent of the writing. This letter size-larger than that in normal handwriting but still compatible with fluent action - was imposed to improve the accuracy of the recording, which was important in view of the subsequent numerical computations. No constraint was imposed on horizontal spacing. The individual was asked to write as naturally and clearly as possible, as teachers do in grade school. For each trigram, a set of 100 traces was recorded in close temporal succession, beginning with lll.

Handwriting traces were processed as follows. After digital lowpass filtering (cutoff, $10 \mathrm{~Hz}$ ), we isolated the middle $l$ in the trigrams, using the minima of the tangential velocity as landmarks, and we measured the durations of the upstroke $\left(t_{\mathrm{u}}\right)$ and downstroke $\left(t_{\mathrm{d}}\right)$ of this letter (the first $l$ was added to minimize the influence of the settling-in phase of the movement). For each trigram, a set of 10 traces of the middle $l$ (templates) were selected among all available records, using two criteria: (1) The shape of the template in $l l l$ was as similar as possible to that in $l l n$, and (2) the variance of both $t_{\mathrm{u}}$ and $t_{\mathrm{d}}$ was minimum. The criteria were applied simultaneously as follows. First, we computed the average $l$-trace for both trigrams. Because traces had different durations, averages were computed after interpolating the original samples (25-harmonics Fourier) and normalizing them to a constant number of samples. Second, we ranked all the $l$-traces for each trigram according to their least square distance from the average trace in the other trigram. Third, we computed the variances of $t_{\mathrm{u}}$ and $t_{\mathrm{d}}$ over each possible ranked set of 10 traces (i.e., [1-10], [2-11], .. [91-100]). Finally, we selected the two sets that minimized the weighted combination of the distance and variance criteria. The average stroke durations for the two sets were: $l l l, t_{\mathrm{u}}=256.0 \mathrm{msec}( \pm 6.15), t_{\mathrm{d}}=225.5 \mathrm{msec}( \pm 6.45)$; $\ln , t_{\mathrm{u}}=309.5 \mathrm{msec}( \pm 6.00), t_{\mathrm{d}}=387.5 \mathrm{msec}( \pm 7.9)$. The average proportions of the total duration $\left[r=t_{\mathrm{d}} /\left(t_{\mathrm{u}}+t_{\mathrm{d}}\right)\right]$ were: $l l l, r=.468$; $l n, r=.555$. The average velocities of the strokes were: $l l l, V_{\mathrm{u}}=$ $29.95 \mathrm{~cm} / \mathrm{sec}, V_{\mathrm{d}}=28.07 \mathrm{~cm} / \mathrm{sec} ; l l n, V_{\mathrm{u}}=24.14 \mathrm{~cm} / \mathrm{sec}, V_{\mathrm{d}}=$ $16.77 \mathrm{~cm} / \mathrm{sec}$. Overall, the average tangential velocity was higher for $l l l(V=29.27 \mathrm{~cm} / \mathrm{sec})$ than for $l n(V=20.01 \mathrm{~cm} / \mathrm{sec})$. The average length of the trajectory was virtually identical in the two sets of $l$-traces ( 13.98 and $13.95 \mathrm{~cm}$, respectively), and so was the average shape. Panels A and B in Figure 1 illustrate the extraction of the template from typical records. Panels $\mathrm{C}$ and $\mathrm{D}$ show the average and the $95 \%$ confidence interval of the trajectory of the templates computed over the selected 10 templates. Panels $\mathrm{E}$ and $\mathrm{F}$ show the average and standard deviation of the corresponding velocity profiles (for ease of comparison with the stimuli, total durations were normalized to $1 \mathrm{sec}$ and velocities were scaled accordingly). In the same panels, the time course of the radius of curvature of the corresponding average trajectory is also shown.

In the second step of the procedure, the kinematics of the stimuli were manipulated. For each template, we generated seven stimuli that differed only in the distributions of the tangential velocity along the trace. The logic of the manipulation was the following. Because the templates were instances of actual writing movements, the tangential velocity of the stimulus $V(s)$ at the curvilinear coordinate $s$ and the corresponding radius of curvature $R(s)$ covaried in a regular fashion that is well captured by the empirical power law (two-thirds power law; Viviani \& Stucchi, 1992):

$$
V(s)=K(s)[R(s) /(1+\alpha R(s))]^{1-\beta} .
$$

In this equation, the parameter $\alpha$ ranges between 0 and .1, depending on the average tangential velocity. $K(s)$ is a term (called velocity gain factor) that does not depend on the form of the trajectory. In most cases, $K(s)$ can be well approximated by a piecewise constant function whose values scale with the length of the corresponding segments of the trajectory (Viviani \& Cenzato, 1985). The distribution of the tangential velocity along the trajectory depends critically on the exponent $\beta$. As is the case for all curvilinear drawing movements, the value of the exponent that yielded the best approximation to the kinematics of the templates was $\beta=2 / 3$.

It has been shown (Viviani \& Stucchi, 1992) that, for any choice of the exponent $\beta$ and of the velocity gain factor $K(s)$, there is a unique transformation $\tau=\mathrm{f}(t)$ of the time scale of the template such that the new set of samples $[x(\tau), y(\tau)]$ satisfies the general power law (Equation 1) with that value of $\beta$ (the transformed set of samples describes the same trajectery as the original one). Moreover, for any time interval $T$, there is a (unique) constant value of $K$ such that the total duration of the movement is $T$. By this procedure, each template was transformed, using seven equally spaced values of $\beta-1 / 6,2 / 6$, $3 / 6,4 / 6,5 / 6,6 / 6$, and $7 / 6$-yielding a total of 2 (trigrams) $\times 10$ (templates) $\times 7(\beta)=140$ stimuli. In all cases, the duration of the movement was set to $T=1 \mathrm{sec}$. Following a calibration procedure detailed in Viviani and Stucchi (1992), the parameter $\alpha$ was set to .05 . Figures $2 \mathrm{~A}$ and $2 \mathrm{~B}$ show the tangential velocity profiles corresponding to the indicated values of $\beta$ for one typical template from each trigram. For any choice of $\alpha, \beta$, and $K$, these profiles depend only on the radius of curvature of the corresponding template. Note that the stimuli for $\beta=4 / 6=2 / 3$ were approximations of the original templates (compare the thick trace labeled $4 / 6$ with the averages in Figures $1 \mathrm{E}$ and $1 F$ ). However, the difference between the first and the second peaks of velocity, present in all the templates for $l l n$, was not accurately reproduced in the stimuli. Matching also this aspect of the templates would have required stimulus-by-stimulus tailoring of a stepwise constant gain function $K(s)$. For reasons to be considered in the Discussion section, it was not necessary to perform this fine-tuning procedure.

The participants were told that they would be shown the dynamic trace of the letter $l$, excerpted from a continuous writing movement. They were informed that the letter after the $l$ in the actual complete movement could be either another $l$ or an $n$. The task was to predict this letter. Trials began by a visual warning message. After a fixed $1-\mathrm{sec}$ interval, the stimulus was traced on the screen by a $2-\mathrm{mm}$ dot. The disappearance of the entire trace at the end of the movement marked the beginning of the response period. No time constraints were imposed, but the participants were encouraged to rely only on their immediate appreciation of the "inertia" of the dot. No performance feedback was given.

There were seven blocks of 20 stimuli, one for each $\beta$ value. Each block included 10 templates for the trigram $l l l$ and 10 for the trigram $l l n$. The 140 trials were administered in a single session, with the order of presentation of the blocks and the order of the stimuli within a block being randomized across participants. The experiment was preceded by an informal demonstration of the procedure and by 10 practice trials. A complete session lasted approximately $30 \mathrm{~min}$. The participants could stop for a short pause after the end of a block.

There were two conditions, each testing one response modality. In the first condition (three-choice), the participants had the option of not answering whenever they did not feel confident about their 

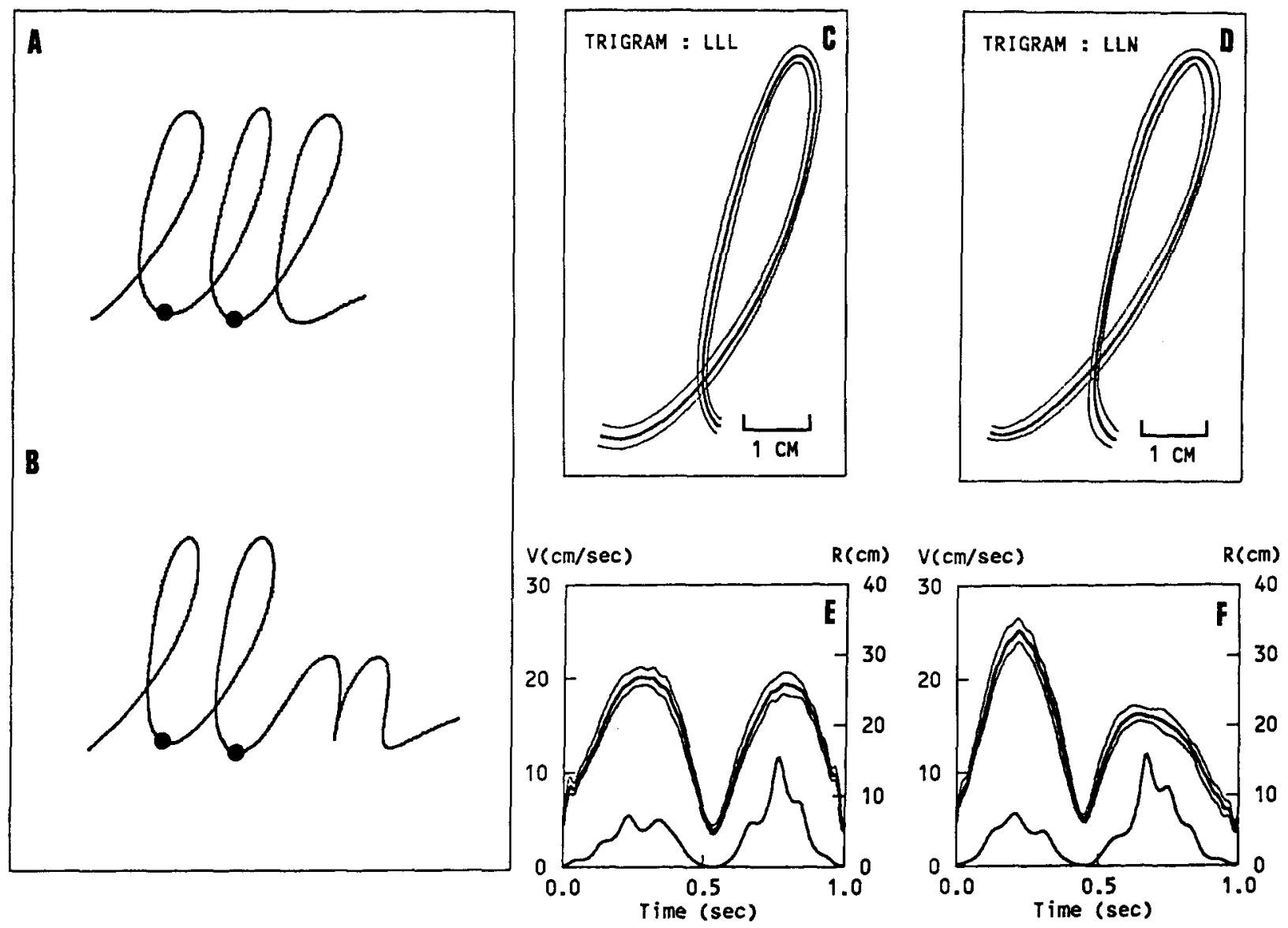

Figure 1. Description of the templates. Panels A and B: one typical trace for each trigram. Dots delimit the templates of the middle l extracted from the traces. Panels C and D: thick lines, average trajectories of the templates for the two trigrams (computed over 10 templates); thin lines, geometrical variability of the traces. Bands around the mean are the envelope of the confidence ellipses for each sample. Because each template had a slightly different duration, averages and confidence ellipses were computed after interpolating the original traces (25-harmonics Fourier) and normalizing to a constant number of samples (500). Panels $E$ and $F$ : average and standard deviation of the tangential velocity as a function of time (computed over the same 10 templates). For ease of comparison with the analogous plots for the stimuli (Figure 2), durations have been normalized to 1 sec, and velocities have been scaled accordingly. Also shown is the corresponding evolution of the radius of curvature of the average trajectory.

judgment. The response was entered by clicking with the mouse on one of three icons, marked " $l$," "In," and "?," corresponding to the two possible continuations and the no answer option, respectively. In the second (forced-choice) condition, the no answer option was suppressed, and only two response icons appeared on the screen. Aside from the difference in response rule, the two conditions were identical. They were tested successively on different groups of participants.

\section{RESULTS}

\section{Three-Choice Response Rule}

The ability to predict the following letter depended on the value of the exponent $\beta$-that is, on the distribution of the tangential velocity along the trace. Figure 3 shows the probabilities (frequencies) of correct, incorrect, and no answer responses as a function of $\beta$ (averages over all participants). For $\beta=4 / 6$ - that is, when the stimuli approximated a real handwriting movement - the accuracy of the prediction was reasonably high (pooling over let- ters, $P\{$ Correct $\}=.69$ ). When the velocity distribution was not too different from the one prescribed by the twothirds power law $(\beta=3 / 6$ and $\beta=5 / 6)$, the drop in accuracy was significant but not dramatic. The pattern for incorrect responses was almost the mirror image of the one for correct responses. The probabilities for the three center values of $\beta$ were well above and below chance level for correct and incorrect responses, respectively (chance level is defined as $(1-P\{$ No Answer $\}) / 2)$.

The probabilities of the three responses were not independent. However, in order to assess the effects of the experimental factors, separate analyses of variance (GLM, repeated measures design) were performed for each response type after applying the arcsin transformation $[w=$ $2 \cdot \arcsin (\sqrt{ } P)]$. The within-subjects factors were the two possible responses ( $l$ or $n$ ) and the exponent $\beta$. The results are reported in Table 1 . The effect of $\beta$ was highly significant for both correct and incorrect responses. A marginal, nonsignificant effect ( $p=.104)$ was present 


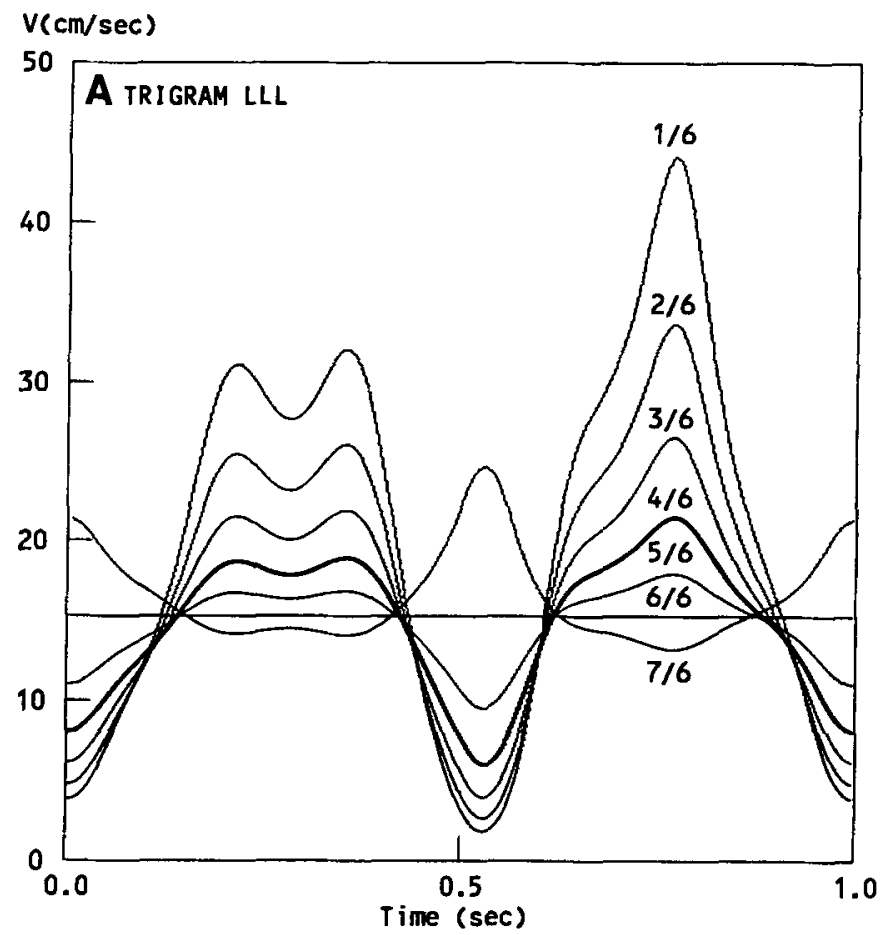

$\mathrm{V}(\mathrm{cm} / \mathrm{sec})$

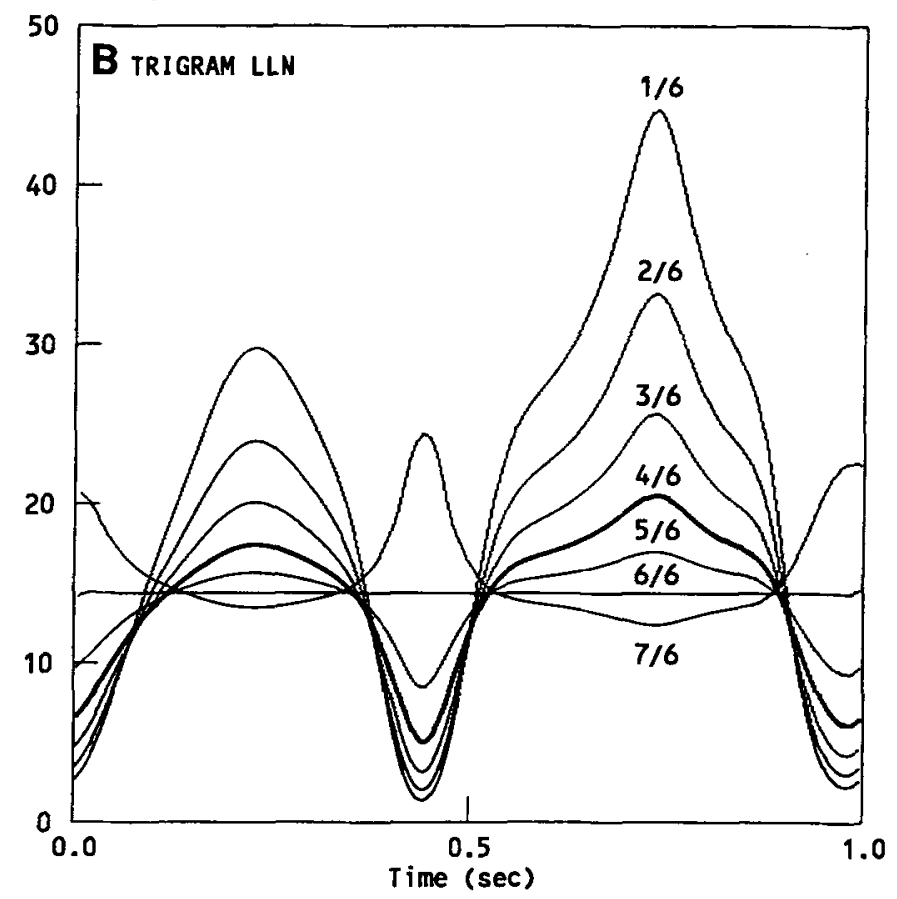

Figure 2. Generation of the stimuli. Each panel illustrates the result of the generation procedure for one typical template from each trigram. In both cases, the tangential velocity of the middle $l$ for the indicated values of $\beta$ is plotted as a function of time. Velocities were computed by inserting the values of the radius of curvature of the template in the twothirds power law. Similar traces were obtained for the other nine templates. The average tangential velocity and the total duration of the stimuli were the same for all $\beta \mathrm{s}$, and so was, therefore, the length of the trajectory. When $\beta=6 / 6=1$, the tangential velocity is constant. When $\beta=7 / 6$, peaks and troughs of the velocity are inverted; the velocity is maximum at the points of highest curvature. Thick lines: velocity profiles that comply with the two-thirds power law $(\beta=4 / 6)$. 
RESPONSE PROBABILITY

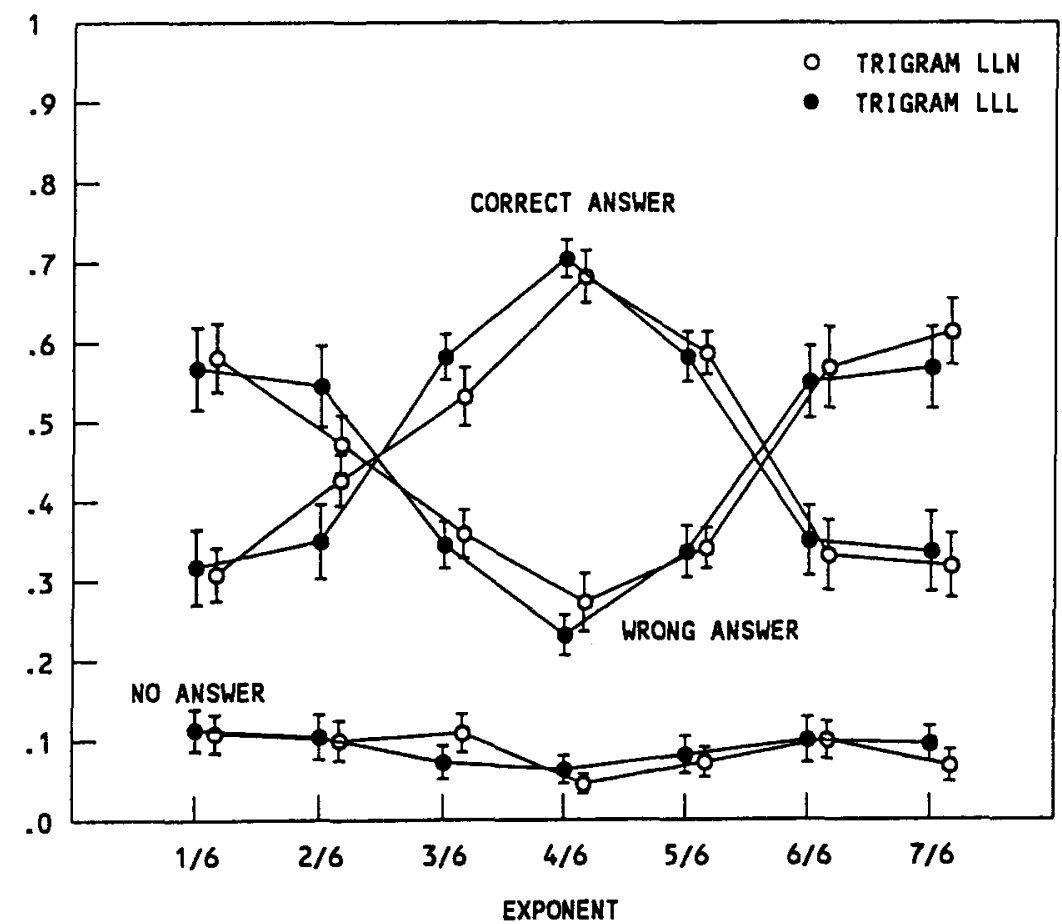

Figure 3. Response probabilities as a function of $\beta$ in the three-choice experiment. Results are averaged over all the participants. Probabilities are estimated from 22 (participants) $\times 10$ (repetitions) $=\mathbf{2 2 0}$ responses (see the text). Bars encompass two standard deviations of the mean individual probabilities. Note the inversion at the extreme values of $\beta$, where wrong answers dominate over correct ones.

also for the no answer response, despite the fact that the corresponding average rate did not exceed $10 \%$. The letter to be guessed was not a significant factor for any response type. We also performed all pairwise comparisons (paired $t$ test) between adjacent $\beta$ values, after collapsing response probabilities over the nonsignificant letter factor. For correct answers, differences were significant at $p<.001$ for the pairs of $\beta$ values [2/6-3/6], [3/6-4/6], [4/6-5/6], and $[5 / 6-6 / 6]$ and significant at $p<.05$ for $[1 / 6-2 / 6]$. None of the differences for pairs of symmetrical $\beta$ values (with respect to $\beta=4 / 6$ ) was significant. An inversion occurred at the extreme values of $\beta$. For three of these values $(1 / 6,6 / 6,7 / 6)$, mistaken predictions were actually significantly more frequent than correct ones (paired $t$ test: $\beta=1 / 6, t_{21}=3.136, p=.005 ; \beta=6 / 6, t_{21}=3.092, p=$ $.006 ; \beta=7 / 6, t_{21}=3.348, p=.003$; pooling over the two letters). Thus, when the velocity profiles differed most from that of the templates, the participants, rather than performing at chance level, actually inverted their responses.

We searched for evidence of learning in the course of the experiment. Because each block confronted the participant with a new condition and because blocks were administered in a different random order to each participant, the search was conducted independently within blocks. For each block (i.e., for each value of $\beta$ ), we computed the probabilities of the three possible responses averaged across participants. Because the results did not differ significantly, these averages were then collapsed over $\beta$ values symmetrical with respect to $\beta=2 / 3$ (Table 2 ). For all values of $\beta$, the variation of the response probabilities as a function of the trial rank order within blocks followed the same simple pattern. The rate of no answer responses decreased during the first few trials, approaching zero at the end of the block. The proportion of mistakes increased pari passu by the same amount, leaving the proportion of correct answers virtually unchanged (linear correlation between $P$ \{correct $\}$ and trial rank order within the block, $r^{2}=.105, p=.362$ ). In conclusion, within-block analysis provided no evidence that repeated exposure to the same condition improved perceptual discrimination. In fact, after the experiment, many of the participants reported the subjective impression of responding at random on most trials, suggesting unawareness of the cues processed during the presentation of the stimuli.

\section{Forced-Choice Response Rule}

The fact that, contrary to expectation, the probability of the no answer response did not increase as $\beta$ moved away from the central $4 / 6$ value may cast doubt over the interpretation that the participants gave to this option. Thus, although the overall frequency with which this option was taken remained low, it was necessary to test 
Table 1

Response Probabilities: Summary of the Analysis of Variance

\begin{tabular}{|c|c|c|c|c|}
\hline Effect & $F$ & $d f$ & $M S_{\mathrm{e}}$ & $p$ \\
\hline \multicolumn{5}{|c|}{ Three-Choice Response Rule } \\
\hline \multicolumn{5}{|l|}{ Correct responses } \\
\hline Letter to be guessed & 0.009 & 1,21 & 0.452 & .925 \\
\hline Exponent $\beta$ & 31.949 & 6,21 & 0.158 & .000 \\
\hline Interaction & 1.291 & 6,126 & 0.107 & .266 \\
\hline \multicolumn{5}{|l|}{ Incorrect responses } \\
\hline Letter to be guessed & 0.079 & 1,21 & 0.449 & .781 \\
\hline Exponent $\beta$ & 23.597 & 6,21 & 0.181 & .000 \\
\hline Interaction & 0.621 & 6,126 & 0.135 & .621 \\
\hline \multicolumn{5}{|l|}{ No response } \\
\hline Letter to be guessed & 0.025 & 1,21 & 0.167 & .875 \\
\hline Exponent $\beta$ & 1.802 & 6,21 & 0.122 & .104 \\
\hline Interaction & 0.647 & 6,126 & 0.173 & .647 \\
\hline \multicolumn{5}{|c|}{ Forced-Choice Response Rule } \\
\hline \multicolumn{5}{|l|}{ Correct responses } \\
\hline Letter to be guessed & 0.097 & 1,21 & 0.008 & .759 \\
\hline Exponent $\beta$ & 107.450 & 6,21 & 0.004 & .000 \\
\hline Interaction & 0.436 & 6,126 & 0.003 & .854 \\
\hline
\end{tabular}

whether such a potential misinterpretation had a significant effect on the selection of the other two responses. The forced-choice response rule experiment was designed for this purpose.

Figure 4 shows the probability of correct answers as a function of the exponent $\beta$ with the same format as that in Figure 3. For both letters, the general trend of the data confirmed fully the results of the three-choice condition. Once again, response accuracy was maximum for $\beta=4 / 6$ and dropped symmetrically for both higher and lower values of $\beta$. Statistical analysis was performed only for correct answers with the same modality as in the first con- dition (Table 1). The analysis revealed a massive effect of the exponent $\beta$, no effect of the letter to be guessed, and no significant interaction between factors. For all $\beta$ values, the probability of a wrong answer remained virtually unchanged with respect to the three-choice condition (averages across $\beta \mathrm{s}, P=.454$ and $P=.458$ for the three-choice and the forced-choice conditions, respectively). Thus, the increase of the average probability of a correct answer (across $\beta \mathrm{s}, .086$ ) matched almost exactly the average no answer probability in the first condition (across $\beta \mathrm{s}, .088$ ).

\section{DISCUSSION}

The experiment confirmed that humans use perceptual information to infer the future course of ongoing dynamic events. Specifically, we documented one instance in which discriminal information, accrued by observing the tracing of one letter in cursive handwriting, is sufficient to predict fairly confidently the next letter. The main result was the demonstration that information pick-up is no longer effective if the tangential velocity does not covary with the curvature of the trace in the way it does in actual writing movements.

To place this result in perspective with respect to previous demonstrations of perceptual anticipation, let us consider the conditions that make such anticipation possible. On the one hand, the observer may tap into his or her knowledge of the rules that are supposed to legislate the unfolding of the perceptual event. For instance, in their study of representational momentum, Freyd and Finke (1984) showed that when a sequence of still pictures suggests the rotation of an object, short-term visual memory of the final orientation of the object is shifted in the di-

Table 2

Response Probabilities: Evolution Within Blocks

\begin{tabular}{|c|c|c|c|c|c|c|c|c|c|c|c|}
\hline \multirow[b]{2}{*}{$\beta$} & \multicolumn{10}{|c|}{ Trial } & \multirow[b]{2}{*}{ Average } \\
\hline & 1 & 2 & 3 & 4 & 5 & 6 & 7 & 8 & 9 & 10 & \\
\hline \multicolumn{12}{|c|}{ Correct Answer } \\
\hline $1 / 6-7 / 6$ & .31 & .31 & .35 & .31 & .35 & .29 & .34 & .29 & .31 & .34 & .29 \\
\hline $2 / 6-6 / 6$ & .37 & .33 & .37 & .35 & .41 & .34 & .35 & .37 & .34 & .40 & .36 \\
\hline $3 / 6-5 / 6$ & .52 & .60 & .57 & .58 & .59 & .57 & .53 & .60 & .57 & .58 & .57 \\
\hline $4 / 6-4 / 6$ & .66 & .64 & .73 & .75 & .68 & .68 & .68 & .68 & .75 & .68 & .69 \\
\hline Average & .47 & .47 & .50 & .50 & .51 & .47 & .48 & .49 & .49 & .50 & \\
\hline \multicolumn{12}{|c|}{ Wrong Answer } \\
\hline $1 / 6-7 / 6$ & .42 & .48 & .48 & .57 & .59 & .66 & .64 & .68 & .67 & .65 & .58 \\
\hline $2 / 6-6 / 6$ & .33 & .38 & .53 & .54 & .50 & .61 & .60 & .55 & .63 & .60 & .53 \\
\hline $3 / 6-5 / 6$ & .27 & .28 & .25 & .34 & .33 & .40 & .42 & .36 & .39 & .41 & .34 \\
\hline $4 / 6-4 / 6$ & .18 & .20 & .25 & .23 & .29 & .27 & .27 & .27 & .25 & .29 & .25 \\
\hline Average & .30 & .34 & .38 & .42 & .43 & .49 & .48 & .46 & .49 & .49 & \\
\hline \multicolumn{12}{|c|}{ No Answer } \\
\hline $1 / 6-7 / 6$ & .27 & .21 & .17 & .12 & .06 & .04 & .02 & .03 & .02 & .01 & .13 \\
\hline $2 / 6-6 / 6$ & .30 & .29 & .10 & .11 & .09 & .05 & .05 & .08 & .03 & .00 & .11 \\
\hline $3 / 6-5 / 6$ & .21 & .12 & .18 & .08 & .08 & .03 & .05 & .04 & .04 & .01 & .09 \\
\hline $4 / 6-4 / 6$ & .16 & .16 & .02 & .02 & .03 & .05 & .05 & .05 & .00 & .03 & .06 \\
\hline Average & .23 & .19 & .12 & .08 & .06 & .04 & .04 & .05 & .02 & .01 & \\
\hline
\end{tabular}

Note - Response probabilities were collapsed over $\beta$ values symmetrical with respect to $\beta=4 / 6$. The number of observations for $\beta=4 / 6$ was half the number of observations for the other paired values of $\beta$. 
RESPONSE PROBABILITY

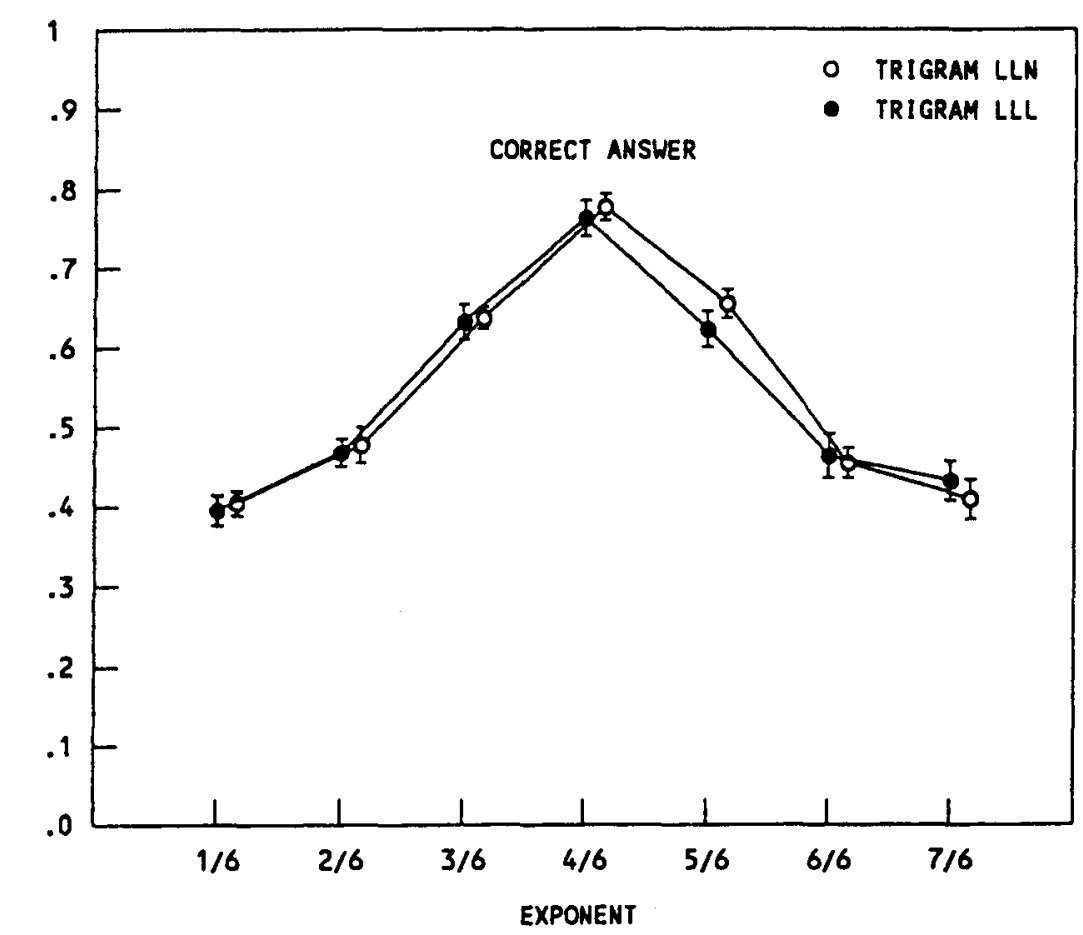

Figure 4. Response probabilities as a function of $\beta$ in the forced-choice experiment. Results are averaged over all the participants. Bars encompass two standard deviations of the mean individual probabilities.

rection of the suggested rotation. According to Finke, Freyd, and Shyi (1986), this phenomenon reflects the tendency to imagine the continuation of the implied motion beyond the end of the inducing sequence: "These extrapolations occur along 'representational pathways' which refer to internalized paths corresponding to the physical paths along which the observer expects the motions to continue" (Finke \& Shyi, 1988, p. 112). In other words, the figures being rotated are implicitly construed as representing real massive objects that comply with the inertial rule that, barring exceptional circumstances, moving objects do not stop cold in midflight. Somehow, this internalized rule generates expectations that interfere with the memory trace.

Knowledge about the likely continuation of the perceptual event appears to be of little use in our experiment, in which the observers had to predict the forthcoming letter by simply watching a small segment of continuous writing. Indeed, even if the traces evoked perceptually the movement of an object under the effect of physical forces, it would be difficult to explain how the observer could envisage two sharply different continuations. It is equally unlikely that the participants took advantage of perceptual cues detected in the course of the experiment, because the tracing of the complete trigram was never shown to the participant and responses were not reinforced. Each block confronted the participants with a novel condition (namely, a new value of $\beta$ ), preventing them from accumulating experience across blocks. In fact, a within-block analysis (Table 2) showed a similar evolution of the response probabilities for all $\beta$ values, with no evidence of improvement in performance across blocks. Instead, the decreasing proportion of no answer responses and the concomitant increase in mistakes suggest that the effect of repetition was simply to make the response strategy less conservative.

On the other hand, we may take advantage of the regularities detected in previous instances of the complete event. For instance, in predicting a forthcoming phonetic segment while we listen to continuous speech, we are likely to take advantage of coarticulatory cues induced backward on previous segments. Similarly, one cannot rule out the possibility that, in our study, the participants exploited purely visual regularities of the handwriting movement identified through past experience. However, it should be stressed that, unlike the case of speech perception, there is a considerable difference between handwriting movements as displayed in this experiment and what we see by looking at someone - or even ourselves-writing.

In order to account for the level of perceptual anticipation demonstrated by the results, we suggest an alternative motor hypothesis, based on the core assumption underlying the motor theory of speech perception (Liberman \& Mattingly, 1985)-namely, that "the objects of speech perception are the intended phonetic gestures of the speaker, represented in the brain as invariant motor 
commands that call for movements of the articulators through certain linguistically significant configurations" (p. 2). We propose to generalize this intuition to the perception of dynamic visual events by assuming that the participants compared the visible trace with the invariant motor commands that they would have executed had they been asked to write either $l l l$ or $l l n$. In other words, discriminal information would be evoked by the stimulus through its interaction with the implicit motor knowledge of the participant. An earlier suggestion along these lines has been advanced to explain the recognizability of distorted pseudo-letters displayed statically (Freyd, 1983). Specifically, the fact that characters drawn with a certain handwriting style were better recognized by observers who had been trained to write with that style has been taken to suggest that handwriting recognition is based on tacit motor knowledge. In essence, we extend this suggestion to the case of dynamic traces by postulating that perceptual anticipation, like recognition, also takes advantage of such motor knowledge.

Support for this hypothesis comes from the main result of the study-namely, the effect of varying the exponent $\beta$. We found that altering the relationship between tangential velocity and curvature present in natural writing movements affected negatively the ability to anticipate the next letter. In fact, large deviations from the natural rule even resulted in a paradoxical inversion of the predictions (Figure 3 ). These findings are in keeping with the hypothesis that discriminal information can be gained by comparing the visual input with an internal motor simulation of the action. Indeed, because the simulation would necessarily respect the natural covariation of tangential velocity and curvature, it would become useless-possibly, even misleading - vis à vis stimuli that systematically violate the covariation rule. The process of turning perceptual cues into useful discriminal information may be construed as a contrast involving distinct simulations of the possible continuations of the movement. Alternatively, one may imagine that the perceptual cues trigger just one motor simulation-namely, the one compatible with the available boundary conditions - and that response selection is biased by the outcome of the simulation. In either case, the fact that responses were all the more accurate in that they were fast and spontaneous, along with the participants' feeling that they were responding at random, indicates that these processes are not conscious.

Assuming the validity of the motor simulation hypothesis, one should ask what aspect of the movement gets compared. There are several reasons to rule out geometric factors (i.e., shape) as a source of discriminal information. (1) The shapes of the templates were extremely similar, both within and between trigrams (Figures $1 \mathrm{C}$ and $1 \mathrm{D}$ ); even the systematic but subtle difference between the radii of curvature of the upstroke and the downstroke remained invariant across trigrams (Figures $1 \mathrm{E}$ and $1 \mathrm{~F}$ ). (2) Whatever small shape difference existed between the middle $l \mathrm{~s}$ in the two trigrams remained invariant across $\beta$ values. (3) When the traces are exact reproductions of the writ- ing movement, shape per se does not account for the ability to guess the next letter (Orliaguet et al., 1997). Thus, the hypothetical comparison with motor simulations has to be based on kinematic criteria. There was a significant difference in the total duration of the templates in the two trigrams (481 vs. $697 \mathrm{msec}$ ), possibly because $l l l$, but not $l l n$, was produced by chaining identical elements. This difference, however, was no longer present in the stimuli (see the Method section). Therefore, the discriminating factor must necessarily pertain to the temporal structure of the trace.

Stroke-velocity distributions of the individual graphemes of the trigram $l l l$ were fairly similar. However, with $l l n$, the anticipated necessity of negotiating a change in the direction of rotation (Van Galen et al., 1986) made the peak velocity of the template downstroke lower than that of the corresponding downstroke in $l l l$ (Figures $1 \mathrm{E}$ and $1 F$ ). At the same time, it is likely that the tendency, present in most hand movements, to complete a unit of motor action in a constant time (Viviani \& Cenzato, 1985) is responsible for the higher tangential velocity of the upstroke. Because the tangential velocity of the stimuli was specified only by the radius of curvature of the templates through the general power law (Equation 1) and because the gain factor $K$ was kept constant throughout the trace (see the Method section), this difference in peak velocities was not reproduced in the stimuli. By contrast, the extrema of velocity and radius of curvature occurred at the same time as in the templates, and the relative durations of the up- and the downstrokes were also exactly the same. Ultimately, the duration ratio was the only discriminating temporal aspect of the stimuli. If guessing the following letter is indeed the result of comparing perceptual evidence with the outcome of a motor simulation, the comparison must involve this ratio.

Because the difference in temporal structure was perceptually salient, the need to invoke the involvement of motor competence may be questioned; one may wonder whether the relative durations of the up- and the downstrokes in the two types of stimuli (i.e., purely perceptual evidence) were not, in fact, sufficient for the observers to make consistent guesses. One argument against this possibility is that, since no performance feedback was given, there was no way for the participants to make the correct association between temporal cues and responses. The second argument is that relative timing remained invariant for all $\beta$ values (see Figure 2). Therefore, the large effect of $\beta$ on response probabilities cannot be explained without also taking into consideration nonperceptual factors.

A striking feature of the results was the paradoxical inversion of the responses when $\beta$ values were either much smaller or much larger than $2 / 3$. Indeed, one would presume that stimuli that deviated much from simulated handwriting should yield minimal discriminal information and, therefore, induce a high proportion of no answer responses. One tentative explanation for this apparent paradox runs as follows. As was argued above, the key 
kinematic invariant for discriminating the trigrams was the invariant duration ratio of the two strokes. If a decision is indeed reached with the contribution of internal motor simulations of the stimuli, $\beta$ values different from the biological reference generate a conflict. The duration ratio of the stimulus suggests an initial (correct) guess. However, its curvature--velocity characteristics are at variance with the expected one (actually, at variance with any simulation). Consequently, we suggest that the effect of this discrepancy is to induce the viewer to reject the initial guess and to opt for the alternative (wrong) answer. Note that, in the first few trials of the blocks $\beta=1 / 6$ and $\beta=$ $7 / 6$, the proportion of no answer responses was quite high, comparable with that of mistakes (see Table 2), indicating that the conflict generates ambiguity but is not necessarily misleading. It does become mostly misleading, however, when the no answer option is either progressively abandoned - possibly, because of frustrationor not available, as in the control, forced-choice condition.

We argued above that deviations of the stimuli from standard handwriting generate a potentially confusing conflict. Yet, not even in the case $\beta=2 / 3$ did stimulus velocity reproduce faithfully that of the templates. By necessity (see the Method section), the velocity profiles carried the inprint of the oscillations present in the radius of curvature. No such oscillations were present in the true velocity profiles. More important, because we kept the gain factor $K$ constant, the peak velocities in the up- and downstrokes did not match those of the templates. Why were these discrepancies not as disruptive for the performance as those induced by changing the value of $\beta$ ? We believe the reason to be the correct balance between acceleration and deceleration phases on which the perceptual naturalness of biological motion hinges crucially (Johansson, 1950). Although not perfect, the stimuli for $\beta=2 / 3$ captured this balance far better than those with extreme values of $\beta$. For instance, the ratio between maximum and minimum velocity ranged between 3 and 5 in the stimuli with $\beta=2 / 3$ and between 2.5 and 5 in the templates. By contrast, for $\beta=1 / 6$, this ratio exceeded 20. Differences in peak accelerations were equally large. Apparently, these differences outweighed those between the templates and the optimal stimuli. It is possible that even better performances would have been obtained by a more accurate tailoring of the stimuli. In view of the results, however, such further refinement was not necessary to achieve the goal of the experiment.

One final remark. All our conclusions are based on an experimental condition involving just one cuing letter and two alternative responses. Surely, in other trigrams, discriminal information (i.e., relative timing) may be more ambiguous and yield less reliable anticipations. Nevertheless, to the extent that we were successful in removing all uncontrolled cues specific to the templates selected for testing, there should be no reason to question the theoretical import of the findings. In fact, the aim of the study was less to assess quantitatively the strength of a motor-perceptual interaction than to establish the reality of the phenomenon. As in single-case neuropsychological studies, the identification of a mechanism in a realistic, albeit specific, condition need not be less robust than an identification based on a larger sample.

\section{REFERENCES}

BeARDSWORTH, T., \& BUCKNER, T. (1981). The ability to recognize oneself from a video recording of one's movements without seeing one's body. Bulletin of the Psychonomic Society, 18, 19-22.

Benguérel, A. P., \& Cowan, H. A. (1974). Coarticulation of upper lip protrusion in French. Phonetica, 30, 41-55.

Bertenthal, B. I., Proffitt, D. R., \& Cutting, J. (1984). Infant sensitivity to figural coherence in biomechanical motion. Journal of Experimental Child Psychology, 37, 213-230.

Bertenthal, B. I., Proffit, D. R., \& Kramer, S. J. (1987). Perception of biomechanical motions by infants: Implementation of various processing constraints. Journal of Experimental Psychology: Human Perception \& Performance, 13, 577-585.

COOPER, L. A. (1992). Mental representation of visual objects and events. In G. d'Ydewalle, P. Eelen, \& P. Bertelson (Eds.), International perspectives on psychological science: Vol. 2: The state of the art (pp. 29-49). Hillsdale, NJ: Erlbaum.

dE'SPERATi, C., \& Viviani, P. (1997). The relationship between curvature and velocity in two-dimensional smooth pursuit eye movements. Journal of Neuroscience, 17, 3932-3945.

Finke, R. A., Freyd, J. J., \& Shyi, G. C.-W. (1986). Implied velocity and acceleration induce transformations of visual memory. Journal of Experimental Psychology: General, 115, 175-188.

Finke, R. A., \& SHYI, G. C.-W. (1988). Mental extrapolation and representational momentum for complex implied motions. Journal of Experimental Psychology: Learning, Memory, \& Cognition, 14, $112-$ 120.

FREYD, J. J. (1983). Representing the dynamics of a static form. Memory \& Cognition, 11, 342-346.

FREYD, J. J. (1993). Five hunches about perceptual processes and dynamic representations. In D. Meyer \& S. Kornblum (Eds.), Attention and performance XIV: Synergies in experimental psychology, artificial intelligence, and cognitive neuroscience (pp. 99-120). Cambridge, MA: MIT Press.

FREYD, J. J., \& FINKE, R. A. (1984). Representational momentum. Journal of Experimental Psychology: Learning, Memory, \& Cognition, 10, 126-132.

Heptulla Chatterjee, S., Freyd, J. J., \& Shiffrar, M. (1996). Configural processing in the perception of apparent biological motion. Journal of Experimental Psychology: Human Perception \& Performance, 22, 916-929.

Johansson, G. (1950). Event perception. Uppsala: Almkvist \& Wiksell. JoHANSSON, G. (1973). Visual perception of biological motion and a model for its analysis. Perception \& Psychophysics, 14, 201-211.

Johansson, G., Von Hofsten, C., \& Jansson, A. (1980). Event perception. Annual Review of Psychology, 31, 27-63.

KANDEL, S. (1995). La perception visuelle de l'anticipation motrice en écriture [Visual perception of motor anticipation in handwriting]. Unpublished doctoral dissertation, Université Stendhal, Grenoble, France.

Kandel, S., Bö̈, L.- J., \& Orliaguet, J.-P. (1993). Visual detection of coarticulatory anticipation or ... Guessing what has not yet been written [Abstract]. In Virtual Reality Annual International Symposium (pp. 148-154). Seattle: IEEE Neural Networks Council.

Kandel, S., Orliaguet, J.- P., \& Bö̈, L.- J. (1994). Visual perception of motor anticipation in the time course of handwriting. In C. Faure, G. Keuss, G. Lorette, \& A. Vinter (Eds.), Advances in handwriting and drawing: A multidisciplinary approach (pp. 379-388). Paris: Europa. 
Kandel, S., Orliaguet, J.-P., \& BoË, L.-J. (1995). Visual perception of motor anticipation in handwriting: Influence of letter size and movement velocity. In B. G. Bardy, R. J. Bootsma, \& Y. Guiard (Eds.), Studies on perception and action III (pp. 347-350). Hillsdale, NJ: Erlbaum.

KENKEL, F. (1913). Untersuchungen über den Zusammenhang zwischen Erscheinungsgroße und Erscheinungsbewegung bei einigen sogenannten optischen Täuschungen [Investigations on the relationship between apparent size and apparent movement in certain so-called optical illusions]. Zeitschrift für Psychologie, 67, 358-449.

KORTE, A. (1915). Kinematoskopische Untersuchungen [Cinematoscopic investigations]. Zeitschrift für Psychologie, 72, 194-296.

Lacquaniti, F., Terzuolo, C. A., \& Viviani, P. (1983). The law relating kinematic and figural aspects of drawing movements. Acta Psychologica, 54, 115-130.

Liberman, A. M., Cooper, F. S., Shankweiler, D., \& StuddertKennedy, M. (1967). Perception of the speech code. Psychological Review, 74, 431-461.

Liberman, A. M., \& MatTingly, I. (1985). The motor theory of speech perception revised. Cognition, 21, 1-36.

OrLIAGUET, J.-P., KANDEL, S., \& Bö̈, L.-J. (1997). Visual perception of cursive handwriting: Influence of spatial and kinematic information on the anticipation of forthcoming letters. Perception, 26, 905-912.

PALMER, S. E. (1978). Fundamental aspects of cognitive representation. In E. Rosch \& B. B. Lloyd (Eds.), Cognition and categorization (pp. 259-303). Hillsdale, NJ: Erlbaum.

PrINZ, W. (1992). Why don't we perceive our brain states? European Journal of Cognitive Psychology, 4, 1-20.

ShIFFRAR, M., \& FREYD, J. J. (1990). Apparent motion of the human body. Psychological Science, 1, 257-264.

ShIFFRAR, M., \& FREYD, J. J. (1993). Timing and apparent motion path choice with human body photographs. Psychological Science, 4, 379384.

Thomassen, A. J. W. M., \& Schomaker, L. R. (1986). Between-letter context effects in handwriting trajectories. In H. S. Kao, G. P. Van Galen, \& R. Hoosain (Eds.), Graphonomics: Contemporary research in handwriting (pp. 253-272). Amsterdam: Elsevier.

Thornton, I. M., Pinto, J., \& Shifrrar, M. (1998). The visual perception of human locomotion. Cognitive Neuropsychology, 15, 535552.
Van Galen, G. P., Muelenbroek, R. G. J., \& Hylkema, H. (1986). On the simultaneous processing of words, letters and strokes in handwriting: Evidence for a mixed linear and parallel model. In H. S. Kao, G. P. Van Galen, \& R. Hoosain (Eds.), Graphonomics: Contemporary research in handwriting (pp. 5-20). Amsterdam: Elsevier.

Viviani, P., Baud-Bovy, G., \& Redolfi, M. (1997). Perceiving and tracking kinesthetic stimuli: Further evidence of motor-perceptual interactions. Journal of Experimental Psychology: Human Perception \& Performance, 23, 1232-1252.

Viviani, P., Campadelli, P., \& Mounoud, P. (1987). Visuo-manual tracking of human bidimensional movements. Journal of Experimental Psychology: Human Perception \& Performance, 13, 62-78.

Viviani, P., \& Cenzato, M. (1985). Segmentation and coupling in complex movements. Journal of Experimental Psychology: Human Perception \& Performance, 11, 828-845.

VIVIANI, P., \& LAISSARD, G. (1996). Motor templates in typing. Journal of Experimental Psychology: Human Perception \& Performance, 22, 417-445.

Viviani, P., \& Mounoud, P. (1990). Perceptuo-motor compatibility in pursuit tracking of two-dimensional movements. Journal of Motor Behavior, 22, 407-443.

VIVIANI, P., \& SCHNEIDER, R. (1991). A developmental study of the relation between geometry and kinematics in drawing movements. Journal of Experimental Psychology: Human Perception \& Performance, 17, 198-218.

VIVIANI, P., \& STUCCHI, N. (1989). The effect of movement velocity on form perception: Geometric illusions in dynamic displays. Perception \& Psychophysics, 46, 266-274.

Viviani, P., \& STUCCHI, N. (1992). Biological movements look uniform: Evidence for motor-perceptual interactions. Journal of Experimental Psychology: Human Perception \& Performance, 18, 603623.

Viviani, P., \& Terzuolo, C. A. (1982). Trajectory determines movement dynamics. Neuroscience, 7, 431-437.

WERTHEIMER, W. (1912). Experimentelle Studien über das Sehen von Bewegung [Experimental studies on the visual perception of movement]. Zeitschrift für Psychologie, 61, 161-265.

(Manuscript received May 4, 1998; revision accepted for publication May 11, 1999.) 